\title{
ACCESO A LA INFORMACIÓN, PARTICIPACIÓN PÚBLICA Y ACCESO A LA JUSTICIA EN MATERIA DE MEDIO AMBIENTE: VEINTE AÑOS DEL CONVENIO DE AARHUS
}

\section{ACCESS TO INFORMATION, PUBLIC PARTICIPATION AND ACCESS TO ENVIRONMENTAL JUSTICE : TWENTY YEARS OF THE AARHUS CONVENTION}

\author{
LUCÍA CASADO CASADO \\ Professora titular de Dret Administratiu \\ Centre d'Estudis de Dret Ambiental (CEDAT) \\ Universitat Rovira i Virgili \\ lucia.casado@urv.cat
}

El pasado 25 de junio de 2018, se han cumplido 20 años de la aprobación del Convenio de la Comisión Económica de las Naciones Unidas para Europa sobre "El acceso a la información, la participación del público en la toma de decisiones y el acceso a la justicia en asuntos ambientales", adoptado en la Conferencia Ministerial "Medio Ambiente para Europa", celebrada en Aarhus (Dinamarca) el 25 de junio de 1998 -más conocido como Convenio de Aarhus-. Sin duda, este Convenio, a través de la articulación de tres pilares fundamentales - acceso a la información, participación pública y acceso a la justicia en materia de medio ambiente-, ha supuesto un paso muy importante y un avance clave hacia la instauración de la democracia ambiental. En él se materializaba, por vez primera en un convenio internacional, el principio 10 de la Declaración de Río sobre el Medio Ambiente y el Desarrollo de 1992, en el que ya se reflejaba la trascendencia en la materia ambiental del acceso a la información, la 
participación ciudadana y el acceso a la justicia y, en especial, se ponía de manifiesto la importancia de la participación ciudadana como instrumento para mejorar la protección del medio ambiente.

A través de una regulación contenida en 22 artículos, este Convenio pretende garantizar los derechos de acceso a la información sobre el medio ambiente, la participación del público en la toma de decisiones y el acceso a la justicia en materia de medio ambiente, "a fin de contribuir a proteger el derecho de cada persona, de las generaciones presentes y futuras, a vivir en un medio ambiente que permita garantizar su salud y su bienestar" (art. 1). Con este fin, tras establecer una serie de definiciones y disposiciones generales (arts. 2 y 3 , respectivamente), regula de forma bastante detallada el acceso a la información ambiental (arts. 4 y 5), la participación del público (arts. 6, 7 y 8) y el acceso a la justicia (art. 9) y contiene también una serie de preceptos (arts. 10 a 22) en los que se contienen determinados aspectos de procedimiento de la Convención, como las reuniones de las Partes, el derecho de voto, las enmiendas, la solución de controversias o la firma, ratificación, aceptación, aprobación y adhesión, y también se establece un interesante mecanismo no contencioso, no judicial y consultivo para revisar el cumplimiento de las disposiciones del Convenio. Se trata del primer acuerdo internacional, con eficacia jurídica vinculante, que otorga derechos a la sociedad civil en materia de acceso a la información, participación pública y acceso a la justicia en materia de medio ambiente con el fin de preservar, proteger y mejorar la calidad del medio ambiente. De ahí su relevancia, pues supone un avance fundamental en el reconocimiento y en el ejercicio efectivo de los derechos de acceso a la información, participación y acceso a la justicia para la protección del medio ambiente.

Este Convenio ha sido ratificado por la Unión Europea, mediante la Decisión del Consejo de 17 de febrero de 2005, y también por España, mediante Instrumento de ratificación de 15 de diciembre de 2004 (publicado en el BOE de 16 de febrero de 2005) y ha influenciado de forma decisiva las regulaciones de esta materia, tanto en el seno de la Unión Europea (Directivas del Parlamento Europeo y del Consejo 2003/4/CE, de 28 de enero de 2003, relativa al acceso del público a la información medioambiental; y 2003/35/CE, de 26 de mayo de 2003, que establece medidas para la participación del público en la elaboración de 
determinados planes y programas relacionados con el medio ambiente y por la que se modifican, en lo que se refiere a la participación del público y el acceso a la justicia, las Directivas 85/337/CEE y 96/61/CE del Consejo), como en España (en la actualidad, la Ley $27 / 2006$, de 18 de julio, por la que se regulan los derechos de acceso a la información, de participación pública y de acceso a la justicia en materia de medio ambiente, que sustituye a la anterior Ley 38/1995, de 12 de diciembre, sobre el derecho de acceso a la información en materia de medio ambiente). Es evidente el impacto positivo que la aplicación de esta norma internacional ha tenido en el ordenamiento jurídico de la Unión Europea y en el español, habida cuenta de que ha implicado un avance fundamental en el reconocimiento y en el ejercicio efectivos de los tres pilares de la democracia ambiental. Sin embargo, también son muchas las dificultades que su aplicación efectiva está encontrando. De ahí la conveniencia de realizar una reflexión, a modo de balance, sobre este instrumento internacional cuando se cumple su vigésimo aniversario.

Por otra parte, el mismo año en que se cumple este aniversario, ha visto la luz, tras un largo proceso de gestación que ha durado siete años, el Acuerdo Regional sobre el Acceso a la Información, la participación pública y el acceso a la justicia en asuntos ambientales en América Latina y el Caribe, adoptado el 4 de marzo de 2018 en Escazú (Costa Rica) —más conocido como Acuerdo Escazú-, de cuya adopción debemos congratularnos. En efecto, se ha conseguido un instrumento de naturaleza vinculante, que va a suponer un hito de gran importancia, para los países de América Latina y el Caribe. De ahí la relevancia, también, de analizar a fondo este instrumento.

Es en este contexto donde debemos situar este primer número monográfico de la Revista Catalana de Dret Ambiental, cuyo objeto último es realizar un balance de los tres pilares de la democracia ambiental el año en que se cumplen los veinte años desde la aprobación del Convenio de Aarhus. Sin duda, creo que la iniciativa de la dirección de la Revista Catalana de Dret Ambiental de organizar un monográfico con este objeto, además de estar justificada por el contexto señalado, constituye una decisión plenamente acertada. $Y$ debo manifestar mi agradecimiento por haberme confiado la coordinación de este número. 
Para dar cumplimiento a las finalidades señaladas, el presente número monográfico recoge 5 contribuciones. En cuanto a su contenido, todas ellas se plantean, en diferentes ámbitos territoriales (Europa y, en particular, los Estados miembros de la Unión Europea; España; y América Latina y el Caribe), los avances que ha supuesto para la democracia ambiental la aprobación en dichos territorios de las diferentes normas reguladoras de los derechos de acceso a la información, la participación pública y el acceso a la justicia en materia de medio ambiente (el Convenio de Aarhus; las Directivas 2003/4/CE del Parlamento Europeo y del Consejo, de 28 de enero de 2003, y 2003/35/CE del Parlamento Europeo y del Consejo, de 26 de mayo de 2003; la Ley 27/2006, de 18 de julio; y el Acuerdo Escazú). Sin embargo, todas estas contribuciones tienen un valor añadido. Lejos de limitarse a señalar los logros que han supuesto las diferentes normas mencionadas, que refuerzan la implementación del principio 10 de la Declaración de Río, todas ellas analizan en profundidad las principales debilidades detectadas y los retos todavía pendientes para conseguir una eficaz aplicación de los tres pilares de la democracia ambiental y realizan consideraciones relevantes sobre las perspectivas que se abren y el camino a seguir en un futuro para reforzar la normativa vigente y garantizar una aplicación efectiva de la misma. Estamos, por tanto, ante interesantes balances del estado de la cuestión, que pueden tener una extraordinaria utilidad pro futuro, al incorporar algunas propuestas de lege ferenda y proponer interesantes medidas que podrían suponer un refuerzo importante de los tres pilares que sustentan la democracia ambiental.

El primer trabajo, a cargo de Ludwig Krämer, ("Citizens rights and administrations' duties in environmental matters: 20 years of the Aarhus Convention"), destaca las principales virtualidades del Convenio de Aarhus y analiza exhaustivamente el impacto de los tres pilares contenidos en el mismo —acceso a la información, participación del público y acceso a la justicia-, que han tenido un éxito y una materialización práctica desigual. En tanto que el primero ha tenido un gran éxito, el segundo y el tercero muestran un progreso bastante limitado. Así, realiza una presentación de sus principales logros y omisiones y carencias en cada uno de los tres pilares, especialmente en el seno de la Unión Europea. En el ámbito del acceso a la información, destaca las 
reticencias que todavía tienen muchas autoridades públicas para proporcionar información ambiental; los déficits que presenta la difusión activa de información sobre el medio ambiente; la ausencia de un sistema efectivo de sanciones administrativas o penales contra las autoridades públicas que no cumplan con sus obligaciones; o los problemas para acceder a la información ambiental en manos de algunas instituciones comunitarias. En materia de participación, se hace eco del poco éxito de la participación pública en la toma de decisiones ambientales y de las limitadas mejoras en los procesos participativos, que no han cambiado de forma significativa con el Convenio de Aarhus, al tiempo que plantea cómo la participación pública en la adopción de decisiones relacionadas con el medio ambiente podría mejorarse y ampliarse mucho más allá de las disposiciones de este Convenio. En cuanto al acceso a la justicia, también destaca las escasas mejoras innovadoras producidas, dadas las grandes barreras todavía existentes para que un ciudadano individual o una organización ambiental pueda llevar a los contaminadores privados ante los tribunales.

El segundo ("El acceso a la información en materia de medio ambiente en España: balance y retos de futuro"), elaborado por José Antonio Razquin, se centra en el estudio de la regulación específica del acceso a la información ambiental en España, tanto en su faceta activa (recogida y difusión) como pasiva (derecho de acceso previa solicitud), establecida, en aplicación del Convenio de Aarhus, por la Ley 27/2006, de 18 de julio, que supuso un cambio de rumbo hacia la transparencia ambiental. Se analiza este régimen especial y se realiza un balance de la normativa vigente y de su aplicación, mostrando los principales avances y dificultades en la efectividad del acceso a la información ambiental; y también se aborda su relación con la posterior regulación general del acceso a la información pública en la Ley 19/2013, de 9 de diciembre, de transparencia, acceso a la información pública y buen gobierno. A la vista de este balance, el autor ofrece interesantes propuestas de mejora en aras de una amplia y efectiva transparencia en materia de medio ambiente. Entre ellas, destacan las siguientes: la necesaria configuración del acceso a la información ambiental como un derecho fundamental; la exigencia de una adecuada organización de la información ambiental que permita la sencilla y ágil accesibilidad a ella por el público; la necesidad de avanzar en la accesibilidad, interoperabilidad, calidad y 
amplitud de la difusión de la información por las autoridades públicas; la mejora de la regulación del derecho de acceso a la información ambiental mediante solicitud, con una revisión legal y aplicativa de las excepciones; y una protección eficaz del acceso a la información ambiental mediante un recurso especial, con el fin de reparar el punto más débil de la regulación específica del acceso a la información ambiental en España.

En la tercera contribución ("La aplicación de las disposiciones del segundo pilar del Convenio de Aarhus en España. Los avances y los viejos retos"), Carmen Plaza aborda el segundo pilar del Convenio de Aarhus -la participación pública en materia de medio ambiente- y su aplicación en España. En particular, expone los avances que, en ejecución de este segundo pilar del Convenio, ha experimentado el Derecho español, prestando especial atención a las novedades que se han producido en los últimos años y a algunos de los problemas y retos que se plantean hoy para alcanzar una participación pública efectiva. Retos que, en cierto modo, como señala la propia autora, siguen siendo los de siempre. Para ello, tras analizar algunas cuestiones generales sobre el segundo pilar del Convenio de Aarhus, el Derecho de la Unión y su aplicación en España, se centra fundamentalmente en su ejecución normativa a través de las disposiciones de la Ley 27/2006 y algunas resoluciones judiciales especialmente relevantes en su aplicación, así como los informes de cumplimiento presentados hasta la fecha por el Estado español a las reuniones de las Partes del Convenio y las resoluciones adoptadas por su Comité de Cumplimiento en relación con España. A la vista de este análisis, se concluye que, si bien el ordenamiento español ha experimentado importantes mejoras en la regulación de la participación pública en materia de medio ambiente, todavía se está lejos de alcanzar el objetivo de una participación real y efectiva de la sociedad en la definición y gestión de los intereses generales que afectan a nuestro modelo de desarrollo.

Ángel Ruiz de Apodaca, en el cuarto trabajo ("El acceso a la justicia ambiental a nivel comunitario y en España veinte años después del Convenio de Aarhus"), se centra en la regulación y aplicación en España del tercer pilar del Convenio de Aarhus —el acceso a la justicia en materia de medio ambiente-, que es el menos desarrollado legalmente, tal y como evidencia la ausencia de normativa 
de la Unión Europea sobre el mismo y su escueta regulación en la legislación básica estatal. Tras realizar un amplio y detallado análisis de las previsiones del Convenio de Aarhus sobre el acceso a la justicia ambiental, de la concreción de este derecho por la jurisprudencia del Tribunal de Justicia de la Unión Europea y de las previsiones de la legislación básica estatal y de la legislación autonómica en esta materia, pone de manifiesto los principales obstáculos en el acceso a la justicia ambiental, que siguen siendo los mismos veinte años después y principalmente de carácter económico. Entre ellos, destaca la asunción de las costas procesales, el necesario acceso a la justicia gratuita y la batalla de las medidas cautelares. Asimismo, propone algunas soluciones interesantes que podrían fomentar los recursos en defensa del medio ambiente: la instauración de un régimen especial de condena en costas; la eliminación de las tasas y depósitos judiciales; el establecimiento de una regulación especial de las fianzas para la adopción de medidas provisionales o para la ejecución provisional de sentencias. En definitiva, como señala el propio autor, las propuestas para lograr una defensa ambiental adecuada van en el sentido de superar las claras limitaciones económicas que se les plantean en no pocos casos a las ONGs ambientales para un eficaz acceso a la justicia ambiental.

Finalmente, la última contribución ("El Acuerdo Escazú: la implementación del principio 10 de Río en América Latina y el Caribe"), a cargo de Gastón Alejandro Médici, se centra en el análisis del reciente Acuerdo Regional sobre el Acceso a la Información, la participación pública y el acceso a la justicia en asuntos ambientales en América Latina y el Caribe. Se trata del primer estudio publicado en España sobre este acuerdo regional. De ahí su interés. En él podrá encontrar el lector una presentación detallada del contenido de este acuerdo, a través de un examen de sus disposiciones más relevantes. Además de la regulación de los tres pilares, en este Acuerdo, el autor también destaca otros aspectos positivos del mismo, como la introducción de una disposición específica relativa a la tutela de los defensores ambientales, la prohibición de reservas y la consagración de ciertos principios relevantes como el de no regresión. Pero el autor va mucho más allá, habida cuenta de que también ofrece un amplio análisis del camino recorrido por los Estados de la región, a través de un repaso por las diferentes reuniones de negociación, entre 2011 y 2018, que condujeron a la 
materialización de la adopción del acuerdo; y plantea hasta qué punto y con qué alcance el Convenio de Aarhus ha sido un referente principal para este acuerdo regional, señalando las principales similitudes y diferencias entre ambos instrumentos. Asimismo, realiza interesantes consideraciones sobre la relevancia de este acuerdo, las perspectivas que abre en la región y el camino a seguir en un futuro.

Como puede fácilmente apreciarse a la vista de los temas tratados en el presente número monográfico, nuestra pretensión es triple: dar a conocer el recientemente aprobado Acuerdo Escazú y la regulación de los tres pilares en él contenida; realizar un balance del impacto de los tres pilares del Convenio de Aarhus, en particular, en el seno de la Unión Europea; y profundizar en el examen de la regulación de los derechos de acceso a la información, la participación pública y el acceso a la justicia en materia de medio ambiente en España, realizando un balance de su aplicación y destacando los retos todavía pendientes.

Este monográfico no se limita, pues, a un análisis meramente descriptivo de la normativa internacional, europea y española en esta materia, sino que es mucho más ambicioso. Por este motivo, en él podrán encontrarse referencias a los principales estudios doctrinales publicados hasta el momento; a la jurisprudencia más importante recaída en la materia; a las resoluciones del Comité de Cumplimiento del Convenio de Aarhus; y a diferentes informes, de interés en la materia, instrumentos todos ellos de gran importancia, por cuanto aportan importantes pautas jurídicas interpretativas y contribuyen de forma decisiva a fijar el alcance de muchos de los preceptos de la normativa vigente en este ámbito. Asimismo, en todo momento, los autores mantienen una perspectiva crítica y, lejos de mencionar sólo los hitos y los logros, también destacan aquellos puntos débiles de la regulación y aplicación de los derechos de acceso a la información, la participación pública y el acceso a la justicia y señalan los aspectos que podrían ser susceptibles de mejora en futuras regulaciones, con el fin de reforzar la efectividad práctica de estos derechos y dotar su aplicación de mayores garantías.

Este planteamiento es, en mi opinión, el gran acierto de los trabajos incorporados en este monográfico. Más allá de recoger los méritos y de los logros asociados 
a la aprobación del Acuerdo Escazú y del Convenio de Aarhus - y a las normas que lo han implementado en la Unión Europea y en España—, aportan otra visión de los mismos, muy crítica, que no repara en destacar todos los problemas que plantean estas regulaciones y señalar todas las dificultades con que se encuentra su aplicación efectiva. El Convenio de Aarhus aparece, así, en su vigésimo aniversario, como un convenio con luces, que sigue siendo uno de los principales instrumentos jurídicos para la participación de la ciudadanía en la defensa del medio ambiente y el acceso a la justicia ambiental, pero también con muchas sombras. $\mathrm{Si}$, en teoría, es un gran instrumento para introducir la democracia ambiental y permitir a los ciudadanos proteger el medio ambiente, en la práctica, lamentablemente, la realidad no ha sido así y su aplicación no se ha llevado a cabo de una forma totalmente adecuada y con los medios necesarios para su efectividad. Tras veinte años de su aprobación y a pesar de los avances producidos, el espíritu contenido en el mismo todavía no constituye una realidad efectiva en el día a día de la defensa ambiental en muchos países -entre ellos, España-. A pesar del reconocimiento formal de determinados derechos relacionados con el acceso a la información, la participación pública y el acceso a la justicia en materia ambiental, todavía no existe una verdadera cultura participativa y de transparencia y tampoco se garantiza suficientemente el acceso a la justicia en cuestiones ambientales y, por ende, la defensa ante los tribunales de los intereses ambientales. España, y también otros países de la Unión Europea, tienen todavía muchos deberes pendientes para lograr la aplicación efectiva del Convenio de Aarhus.

Igualmente, este monográfico pretende contribuir a dar difusión de los derechos de acceso a la información, participación pública y acceso a la justicia, tanto para la ciudadanía como para las propias autoridades públicas encargadas de su aplicación. Si bien la regulación de estos derechos es muy reciente en el ámbito de América Latina y el Caribe, en el seno de la Unión Europea y de España, la regulación - muy completa y avanzada - fue adoptada ya hace muchos años. Ahora bien, lo cierto es que todavía resulta una regulación en muchas ocasiones desconocida, tanto para la ciudadanía como para las propias autoridades públicas encargadas de su aplicación y que deben canalizar y dar respuesta a las peticiones de los ciudadanos en este ámbito; y adolece de algunos problemas 
de aplicación que dificultan su efectividad práctica. Por ello, pretendemos, una vez más, insistir en dar a conocer esta normativa, en señalar los principales problemas de aplicación que presenta y en realizar propuestas que supongan una mejora de su regulación y de su aplicación efectiva. Todo ello con el fin de conseguir que la normativa existente se traduzca también en una aplicación efectiva de los tres pilares y se materialice en una verdadera democracia ambiental. El vigésimo aniversario del Convenio de Aarhus ofrece un motivo perfecto para plantear estas reflexiones.

De esta forma, desde el Centre d'Estudis de Dret Ambiental de Tarragona (CEDAT), damos continuidad a una línea investigadora que seguimos desde hace varios años en este ámbito y que se ha materializado en numerosas publicaciones de sus miembros. Además, realizamos un nuevo balance de la aplicación del Convenio de Aarhus, a los 20 años de su aprobación, que se une al que ya realizamos en 2008 cuando se cumplió su décimo aniversario y que se materializó en la organización del Seminario Diez años del Convenio de Aarhus: acceso a la información, participación pública y acceso a la justicia en materia de medio ambiente, que se celebró en la Facultad de Ciencias Jurídicas de la Universitat Rovira i Virgili el día 25 de junio de 2008; y en la publicación de la monografía dirigida por Antoni Pigrau Solé, Acceso a la información, participación pública y acceso a la justicia en materia de medio ambiente: diez años del Convenio de Aarhus (Atelier, Barcelona, 2008). Esperamos poder realizar el siguiente balance en 2028, cuando se cumpla el trigésimo aniversario del Convenio de Aarhus y el décimo aniversario del Acuerdo Escazú, confiando en que se hayan solucionado, si no todos, buena parte de los problemas de aplicación detectados actualmente y destacados en este monográfico y que podamos hablar de una auténtica democracia ambiental. 